\title{
How is the iceberg of COVID-19? Results from a rapid literature review
}

\author{
Ghobad Moradi $^{1,2}$, Fatemeh Gholami ${ }^{3}$, Mohammad Aziz Rasouli ${ }^{1} * \mathbb{D}$, Fahimeh Bagheri Amiri ${ }^{4}$, Yousef Moradi ${ }^{1,2} * \mathbb{D}$
}

Received: 15 Dec 2020

Published: 16 Jun 2021

\begin{abstract}
Background: Given the various reports of the clinical spectrum of the disease, the aim of the present study was to determine possible scenarios of Coronavirus 2019 (COVID-19) iceberg using published articles.

Methods: The present study was a rapid review of all international databases, including PubMed (Medline), Scopus, Web of Sciences, Embase, and Cochrane Library from January 1 to October 30, 2020.

Results: In this review, 7 scenarios were considered for COVID-19 iceberg, in which the range of fatality percentage was estimated to be $0.5 \%$ to $7 \%$, the range of asymptomatic cases $1 \%$ to $88.6 \%$, the range of cases with mild symptoms $8 \%$ to $78 \%$, no symptoms 1 $\%$ to $90 \%$, the range of intensive care unit (ICU) admission was $0.5 \%$ to $14.2 \%$, and finally the intubation percentage was estimated to be $0.2 \%$ to $12.2 \%$. The Scenarios Diamond Princess Cruise Ship and Iceland are closer to the reality of the clinical spectrum of COVID-19 around the world, which represent $0.6 \%$ and $0.5 \%$ of deaths, $0.7 \%$ and $1 \%$ of intubations, $2.5 \%$ and $9.7 \%$ of ICU admissions, $1.1 \%$ and $6 \%$ of hospitalizations, $15 \%$ and $31 \%$ of cases with mild symptoms, and finally $56.9 \%$ and $75 \%$ of asymptomatic cases of COVID-19, respectively, which should now be considered as the basis of the clinical knowledge of the disease.

Conclusion: Understanding the clinical spectrum and natural knowledge of the disease and paying attention to asymptomatic or mild-symptom cases can help to make better decisions and develop more effective interventions to control COVID-19.
\end{abstract}

Keywords: Best Estimates, Iceberg, COVID 19, Clinical Spectrum, Natural Knowledge, Rapid Review

Conflicts of Interest: None declared

Funding: Deputy of Research of Kurdistan University of Medical Sciences

*This work has been published under CC BY-NC-SA 1.0 license.

Copyright $\odot$ Iran University of Medical Sciences

Cite this article as: Moradi G, Gholami F, Rasouli MA, Bagheri Amiri F, Moradi Y. How is the iceberg of COVID-19? Results from a rapid literature review. Med J Islam Repub Iran. 2021 (16 Jun);35:77. https://doi.org/10.47176/mjiri.35.77

\section{Introduction}

Coronavirus 2019 (COVID-19) is a severe acute respiratory infection caused by coronavirus 2 (SARS-CoV-2) that was first identified in Wuhan, China, in December 2019. The clinical spectrum of the disease includes asymptomatic cases, mild illness, such as colds, to severe viral pneumonia, leading to acute respiratory distress syndrome, which is potentially fatal. On March 11, 2020, the World Health Organizatio n (WHO) declared the outbreak of COVID-19 as a global pandemic (1-3). As of Decem-

Corresponding author: Mr Mohammad Aziz Rasouli, Rasouli.m.a@muk.ac.ir Dr Yousef Moradi, yousefmoradi211@yahoo.com

1. Social Determinants of Health Research Center, Research Institute for Health Development, Kurdistan University of Medical Sciences, Sanandaj, Iran

2. Department of Epidemiology and Biostatistics, Faculty of Medicine, Kurdistan University of Medical Sciences, Sanandaj, Iran

3. Department of Epidemiology, School of Public Health, Iran University of Medical Sciences, Tehran, Iran

4. Urology and Nephrology Research Center, Shahid Beheshti University of Medical Sciences, Tehran, Iran ber 14, 2020, a total of 220 countries were affected by the disease, with more than 72 million confirmed cases and approximately 1,617,713 deathis (4-6).

Age distribution of the affected population, access to diagnostic and treatment services, disease transmission rate in different communities, behavior and culture of the community, and finally the effectiveness of behavioral, social, and therapeutic interventions are factors affecting COVID-19 and changes in its incidence, prevalence, and

$\uparrow$ What is "already known" in this topic:

The Iceberg model is an epidemiological model that can be very useful in identifying new cases, caring for existing diseases, and planning future care for the disease in different countries and communities. The results of this study can clear up the ambiguities about the clinical spectrum of COVID-19.

\section{$\rightarrow$ What this article adds:}

The Scenarios Diamond Princess Cruise Ship and Iceland are closer to the reality of the clinical spectrum of COVID-19 around the world, which represent $0.6 \%$ and $0.5 \%$ of deaths, respectively. 
mortality (7-9).

With the progression of the disease and the proliferation of published articles, and contrary to the initial data, the results of studies show a high prevalence of asymptomatic cases. In the Middle East, the prevalence of asymptomatic people with COVID-19 has increased from $0 \%$ in the beginning to $28.6 \%$ at present. Some studies in other parts of the world have even reported a prevalence of asymptomatic cases to be up to $80 \%(10-13)$. The Chinese data showed that of the total studied cases of COVID-19, $22.4 \%$ were suspected, $14.6 \%$ symptomatic, and $1.2 \%$ asymptomatic $(14,15)$. The results of different studies show that the spectrum of the disease -hospitalized, asymptomatic, and symptomatic cases, and the percentage of mortality and death - has different values in a way that there is a significant discrepancy in the presented results $(11,16,18)$. The reason for this discrepancy in the study results can be the unknown nature of the disease, the existence of little information about the disease, poor diagnostic tests, limited access to diagnostic tests to identify affected people in different countries, and different percentages of asymptomatic people with COVID-19 in various communities. Some of the various information and scenarios that have been published about COVID-19 have led to excessive fear and panic in communities, wrong attitudes, and misunderstandings about the disease and its clinical knowledge. Effective planning and intervention require accurate information about the clinical spectrum and knowledge of the disease. Since case counts are highly related to the amount of testing done, the iceberg can be used to estimate the total number of cases around the world. Therefore, it is helpful to identify the clinical and natural spectrum of COVID-19 and recognize the iceberg of the disease as a classic epidemiological model. The Iceberg model is an epidemiological model that can be very useful in identifying new cases, caring for existing diseases, and planning future care for the disease in different countries and communities. It is relevant to surveillance, measurement of population health, measuring the burden of illness, screening, needs assessment, health services planning, and much else; for instance, understanding selection bias. It clarifies the relationship between clinical epidemiology, which deals only with the visible part, and population-based epidemiology (19).

When a pandemic of various infectious diseases occurs, the number of cases detected is not always greater than the number of cases detected. This is a true picture of an iceberg. Items that are detected in a timely and correct manner are placed at the top of the iceberg, and items that are not detected are underwater (20).

The results of this study can clear up the ambiguities about the clinical spectrum of COVID-19 and help to better understand the behavior of the virus. Therefore, the aim of this study was to estimate different scenarios of the COVID-19 iceberg as a classic epidemiological model and to determine and propose the final scenario in relation to the disease through a rapid review.

\section{Methods}

\section{Search Strategy and Search Terms}

The electronic and academic databases used in the study were (Medline) PubMed, Embase, and Scopus. To review the gray literature, credible related websites along with MedRxiv articles and reports on COVID-19 from different countries were examined. The review was not registered in PROSPERO and corresponding authors were not contacted due to time constraints.

Keywords used included "2019-nCoV”, "SARS-CoV2", "COVID-19", "Wuhan Coronavirus", "Coronavirus disease 2019”, "2019 Novel Coronavirus", "Case Fatality Rate", "Severe COVID-19 Pneumonia", "Critical COVID-19", "Mild AND Moderate COVID-19 Pneumonia", "Common COVID-19 Pneumonia", "Tracheal”, "Intubation", "Intensive Care Unit", "Symptomatic", "Asymptomatic", "Death", "Observational Studies", "Case-Control studies", "Cohort studies", "Case Reports", "Case Series".

The search strategy specific to each database is presented in Table 1. The time period for searching articles was from January 1 to October 30, 2020. The indicators for searching the studies were estimating the case fatality rate (CFR), determining the estimates of asymptomatic patients, patients with mild symptoms, hospitalized patients without intensive care unit (ICU) admission, patients requiring ICU admission, and intubation for different scenarios. To ensure the correct selection of articles related to the research topic, 2 researchers (M.R. and F.G.H.) were selected to search the studies, and disagreements were shared with a third researcher (Y.M.). To obtain more sources, the list of sources for selected articles was reviewed to find further studies.

\section{Inclusion and Exclusion Criteria}

Due to the fact that studies with different sample sizes were conducted in different countries, studies with larger sample sizes were selected to achieve the best estimate for different scenarios. All conducted studies whose main outcomes were the following were included: expression of mortality rate, duration and frequency of ICU admission, determining the frequency of symptomatic or asymptomatic cases, hospitalization ciases, and severe and mild cases of COVID-19. Articles whose full-text was not available or whose outcome was outside the main outcomes of the study were excluded.

In this study, to select the best model, the case fatality rate reported in various studies; was first monitored. At the beginning of the COVID-19 pandemic, studies were published from China and Italy that reported high CFR around the world. Therefore, each of these studies was considered as a scenario in the present study. After that, other scenarios were designed according to studies published in the world and compared with these 2 main scenarios (China and Italy) in the world. It should be noted that to determine the scenarios in this review, we were considered indicators such as case fatality rate, frequency of hospitalizations, outpatient, intubation, hospitalization in ICU, mild symptoms, and finally asymptomatic cases. Other items for determining scenarios are as follows: 


\begin{tabular}{|c|c|}
\hline Databases & Simple Search Strategy and Syntax \\
\hline PubMed & 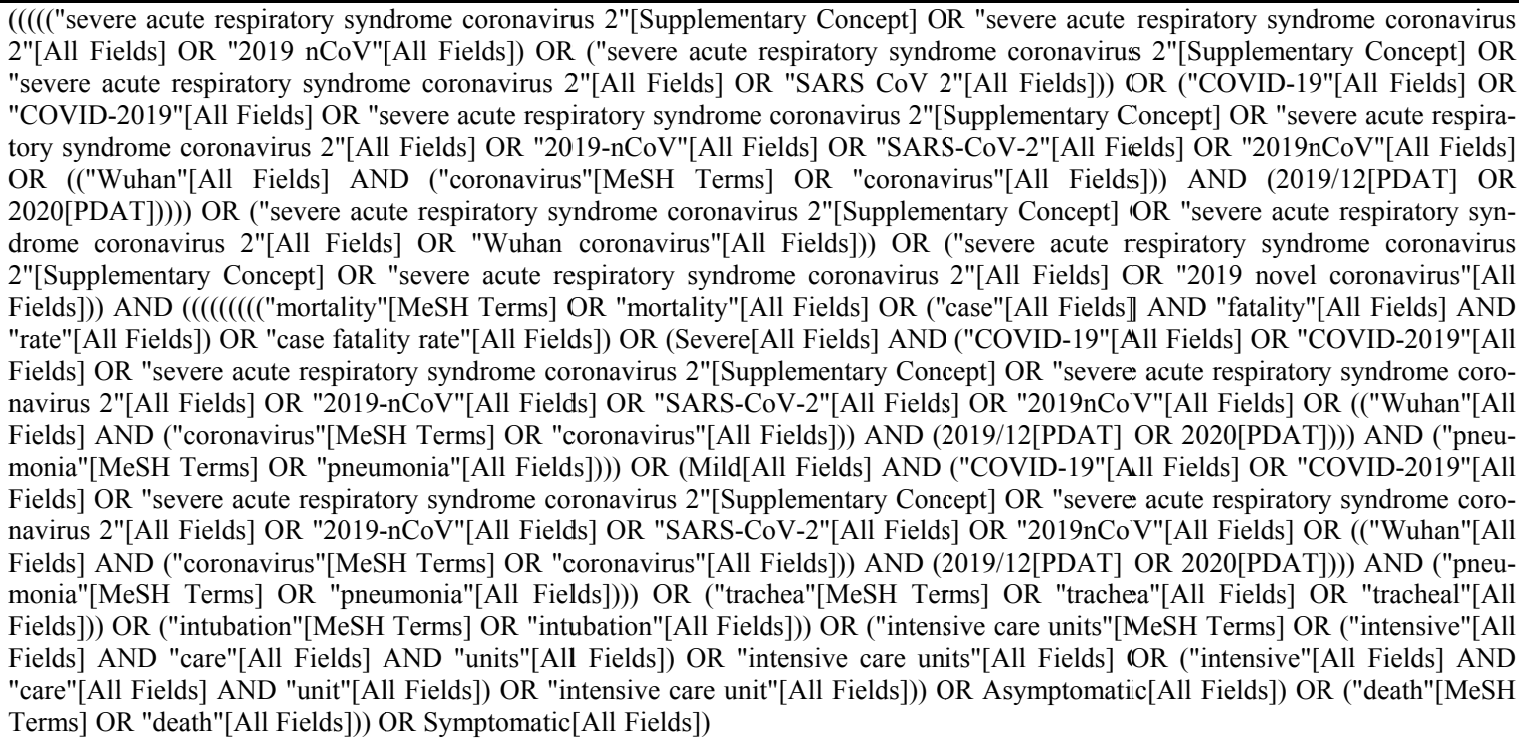 \\
\hline
\end{tabular}

Scopus ( TITLE-ABS-KEY (2019-ncov) OR TITLE-ABS-KEY ( sars-cov-2 ) OR TITLE-ABS-KEY ( covvid-19) OR TITLE-ABSKEY (wuhan AND coronavirus ) OR TITLE-ABS KEY ( coronavirus AND disease 2019) OR TITLE-ABSKEY ( 2019 novel AND coronavirus ) AND TITLE-ABS-KEY ( case AND fatality AND rate ) OR TITLE-ABSKEY ( death) OR TITLE-ABS-KEY ( severe AND covid-19 AND pneumonia) OR TITLE-ABS-KEY ( mild AND covid19 AND pneumonia) OR TITLE-ABS-KEY ( common AND covid-19 AND pneumonia) OR TITLE-ABSKEY ( tracheal) OR TITLE-ABS-KEY ( intubation) OR TITLE-ABS KEY (intensive AND care AND unit) OR TITLE-ABSKEY ( symptomatic ) OR TITLE-ABS-KEY ( asymptomatic ))

Embase ('Wuhan coronavirus' OR 'coronavirus disease 2019'/exp OR '2019 novel coronavirus'/exp) AND ('case fatality rate'/exp OR 'case fatalities' OR 'case fatality' OR 'case fatality rate' OR 'case fatality ratio' OR 'case fatality risk' OR 'se:vere pneumonia'/exp OR 'endotracheal intubation'/exp OR 'intensive care unit'/exp OR 'symptom'/exp OR 'semiology' OR 'symptom' OR 'asymptomatic disease'/exp OR 'asymptomatic condition' OR 'asymptomatic conditions' OR 'asymptomatic disease' OR 'asymptomatic diseases' OR 'asymptomatic disorder' OR 'asymptomatic disorders' OR 'pre-symptomatic condition' OR 'pre-symptomatic conditions' OR 'pre-symptomatic disease' OR 'pre-symptomatic diseases' OR 'pre-symptomatic disorder' OR 'pre-symptomatic disorders' OR 'presymptomatic condition' OR 'presymptomatic conditions' OR 'presymptomatic disease' OR 'presymptomatic diseases' OR 'presymptomatic disorder' OR 'presymptomatic disorders' OR 'death'/exp OR 'death' OR 'mors')

1. Reporting the fatality rate and other indexes using highly accurate real results;

2. The sample size being representative of the community of patients with COVID-19 so that the results can be generalized;

3. Full report of clinical features and symptoms of COVID-19 patients in the results of studies; and

4. Full compliance with diagnostic protocols based on the WHO criteria or other international guidelines for the identification of patients with COVID-19.

In addition, epidemiology logics that focus on the completeness of the study design and reasonable structure studies were considered.

The basis for selecting each of the scenarios is as follows:

Scenario 1. In this scenario, the Italian study was considered because it had examined a large sample size of about 31,506 people and reported a higher fatality rate than other countries at the time of the study $(21,22)$.

Scenario 2. A study published by China was used to design this scenario because its results were the basis for other studies for many months, it had a large sample size, and examined the entire population of Wuhan $(11,23)$.

Scenario 3. A study published in Japan was used to de- sign this scenario. The reason was that it used the agestratified adjustment method in the methodology and findings and calculated and reported changes in known outcomes over time. Using this method significantly reduces the possibility of underreporting. Also, it had a large sample size (24).

Scenario 4. The fourth scenario belongs to South Korea. In this country, appropriate measures, cohort, and serological studies have been conducted on a large sample of infected people with COVID-19 $(n=234,427)(25-27)$.

Scenario 5. This scenario was based on a study conducted in Sweden because it had examined and analyzed the entire affected population of Sweden, so the results could be generalized to the entire population of the country (28).

Scenario 6. The sixth scenario belonged to the studies that were conducted on the Diamond Princess cruise ship. The reason for choosing this scenario was the importance of the patients in this ship and the importance of the ship itself in creating the COVID-19 pandemic and helping to identify ways of infecting the disease during the pandemic (29-33).

Scenario 7. Studies conducted in Iceland were used to design this scenario because they had large sample sizes 
and the results on fatality rate were more reasonable and closer to the world reality $(34,35)$.

\section{Data Extraction}

Our study population included all men and women with COVID-19 without age, racial, and marital status constraints. Given that different studies have reported different values for asymptomatic cases, these values are summarized in 7 general scenarios. The purpose of the study is to present the best estimate in each of the components of the different study pyramids (Table 2). After a meeting with study experts and authors, a checklist was designed to extract the data. It included the author's name, year, and month of publication, country, sample size, age, sex, statistical population, clinical results, frequency of death, hospitalization cases, intubation, and ICU admission, the desired scenario, and reference.

\section{Results}

In this study, 4939 articles remained after conducting an initial search in international databases and removing du- plicates. Then, the articles were screened based on the title and abstract. After this stage, 4869 articles were deleted and 70 articles remained. After reading and fully evaluating the full-text of these 70 articles, 42 were removed because of small sample size and 14 were excluded because they had low clinical information and were less related to the desired outcome. Eventually, 14 articles remained. The final results of this study were prepared and designed based on these articles (Fig. 1). The scenarios' characteristics with other extracted information are reported in $\mathrm{Ta}-$ ble 1 .

After a thorough evaluation of the selected articles in this quick systematic review, several scenarios related to the iceberg phenomenon for the COVID-19 virus were considered in different regions. Using these scenarios, it is possible to determine the fatality rate and other clinical spectrums of COVID-19 in a community.

The first scenario is extracted from the study by Balduzzi, Adriana et al (21) and the Italian National Report (22) (Table 2). These studies were conducted in 2020 in Italy on about 31,506 people with COVID-19. In this sce-

Table 2. The Main Characteristics and Demographic Variables in Included Articles

\begin{tabular}{|c|c|c|c|c|c|c|}
\hline Author & $\begin{array}{l}\text { Country } \\
\text { Years }\end{array}$ & $\begin{array}{l}\text { Sample } \\
\text { size }\end{array}$ & Age, (mean) & Sex & Ice Berge & $\begin{array}{l}\text { Scenario } \\
\text { Number }\end{array}$ \\
\hline $\begin{array}{l}\text { Balduzzi, Adriana et al (21) } \\
\text { Italian National Report (22) }\end{array}$ & $\begin{array}{l}\text { Italy } \\
2020\end{array}$ & 31506 & All population & Both & $\begin{array}{c}\text { CFR }(7 \%) \\
\text { Intubation }(5 \%) \\
\text { ICU }(12 \%) \\
\text { Hospitalized }(13 \%) \\
\text { Mild }(55 \%) \\
\text { No symptom }(8 \%)\end{array}$ & 1 \\
\hline $\begin{array}{l}\text { Wu, Zunyou et al (11) } \\
\text { Guan, X. et al (23) }\end{array}$ & $\begin{array}{l}\text { China } \\
2020\end{array}$ & 73904 & All population & Both & $\begin{array}{c}\text { CFR }(2.3 \%) \\
\text { Intubation }(3 \%) \\
\text { ICU }(6 \%) \\
\text { Hospiitalized }(9.7 \%) \\
\text { Mild }(78 \%) \\
\text { No symptom }(1 \%)\end{array}$ & 3 \\
\hline Russell, Timothy W et al (24) & $\begin{array}{c}\text { Japan } \\
2020\end{array}$ & 3711 & All population & Both & $\begin{array}{c}\text { CFR }(1.9 \%) \\
\text { Intubation }(4.1 \%) \\
\text { ICU }(9 \%) \\
\text { Hospitalized }(30 \%) \\
\text { Mild }(10 \%) \\
\text { No symptom }(45 \%)\end{array}$ & 4 \\
\hline $\begin{array}{l}\text { Jung, Chan-Young et al (25) } \\
\text { Oh, Juhwan et al (26) } \\
\text { Cho, Soo Ick et al (27) }\end{array}$ & $\begin{array}{c}\text { South } \\
\text { Korea } \\
2020\end{array}$ & 234427 & All population & Both & $\begin{array}{c}\text { CFR }(1.8 \%) \\
\text { Intubation }(2.1 \%) \\
\text { ICU }(3 \%) \\
\text { Hospiitalized }(8.7 \%) \\
\text { Mild }(22.4 \%) \\
\text { No symptom }(62 \%)\end{array}$ & 5 \\
\hline Sjodin, Henrik et al (28) & $\begin{array}{l}\text { Sweden } \\
2020\end{array}$ & 16000 & All population & Both & $\begin{array}{c}\text { CFR }(1.5 \%) \\
\text { Intubation }(2.5 \%) \\
\text { ICU }(6 \%) \\
\text { Hospitalized }(18 \%) \\
\text { Mild }(12 \%) \\
\text { No symptom }(60 \%)\end{array}$ & 7 \\
\hline $\begin{array}{l}\text { Yamagishi, Takuya et al (31) } \\
\text { Russell, TW et al (32) } \\
\text { Yamahata, Yoshihiro et al (33) } \\
\text { [Diamond Princess cruise ship] }\end{array}$ & $\begin{array}{c}\text { Japan } \\
2020\end{array}$ & 3714 & All population & Both & $\begin{array}{c}\text { CFR }(0.6 \%) \\
\text { Intubation } 0.7 \%) \\
\text { ICU }(9.7 \%) \\
\text { Hospitalized }(1.1 \%) \\
\text { Mild }(31 \%) \\
\text { No symptom }(56.9 \%)\end{array}$ & 8 \\
\hline $\begin{array}{l}\text { Simon P (35) } \\
\text { Stock, James H et al (34) }\end{array}$ & $\begin{array}{l}\text { Iceland } \\
2020\end{array}$ & 21000 & All population & Male/Female & $\begin{array}{c}\text { CFR }(0.5 \%) \\
\text { Intubation }(1 \%) \\
\text { ICU }(2.5 \%) \\
\text { Hospitalized }(6 \%) \\
\text { Mild }(15 \%) \\
\text { No symptom }(75 \%)\end{array}$ & 9 \\
\hline
\end{tabular}




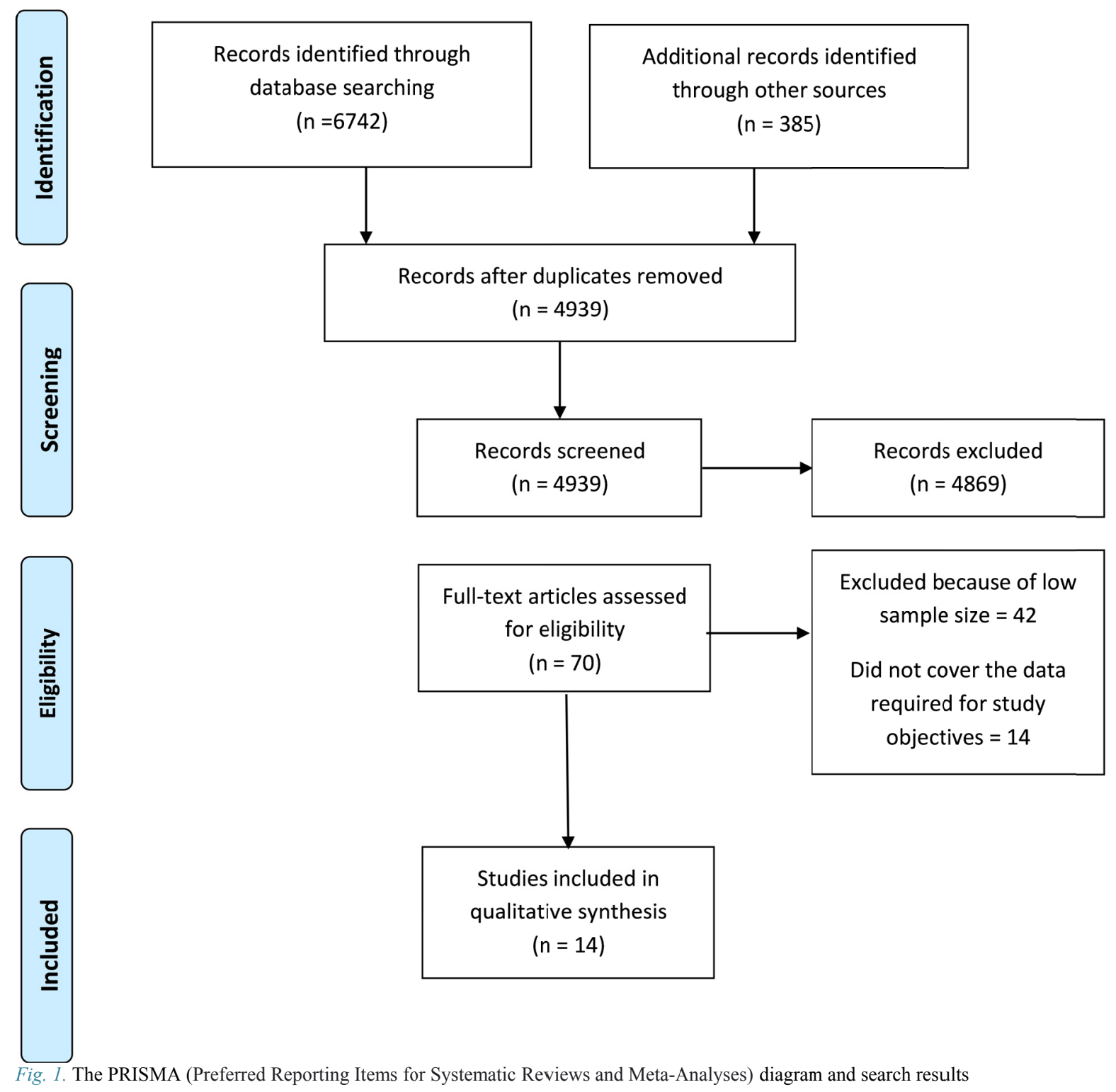

nario, $8 \%$ of cases were asymptomatic and the highest CFR was reported to be $7 \%$ (Fig. 2). The results of this scenario show that $8 \%$ and $55 \%$ of COVID-19 cases were asymptomatic and had mild symptoms of the disease, respectively; $13 \%$ were hospitalized and $12 \%$ were admitted to ICU; and 7\% died (Fig. 2, Tables 2 and 3).

In the second scenario, which was related to the early stages of the disease in China, the results of 2 studies by $\mathrm{Wu}$, Zunyou et al (11) and Guan, X. et al (23) were extracted (Table 2). Some information was extracted from Zunyou et al study and some others from Guan et al study to complete the scenario. Cases without clinical symptoms in this scenario were $1 \%$. Hospitalized cases were $9.7 \%$, $3 \%$ of whom required intubation. Finally, the CFR was reported to be $2.3 \%$ (Fig. 2 and Table 3 ).

The third scenario is considered based on a study by Russell, Timothy W et al (24) conducted in March 2020 in Japan. The sample size in this study was 3711 people (Table 2). In the fourth scenario, $45 \%$ of cases were asymptomatic, more cases were hospitalized (30\%), more cases were admitted to ICU (9\%), and intubation was $4.1 \%$. The more cases are diagnosed, the lower the fatality rate (Fig. 2 and Table 3 ).

The fourth scenario belongs to South Korea (25-27). The studies in this scenario analyzed a total of 234,427 people with COVID-19. In this scenario, the CFR was estimated $1.8 \%$. Also, the frequency of asymptomatic and symptomatic cases was $62 \%$ and $22.4 \%$, respectively. The frequency of intubation, hospitalization, and ICU cases were $2.1 \%, 8.7 \%$, and $3 \%$, respectively (Fig. 2, Tables 2 and 3 ).

The fifth scenario was designed based on the results of studies conducted in Sweden (Sjodin, Henrik et al (28) (Table 2). In the fourth scenario, about $60 \%$ of cases are reported to be without clinicial symptoms, more severe cases are diagnosed, and a percentage of them are hospitalized, older people or cases with underlying disease are admitted to the ICU, and intubation is performed for them. In this scenario, $12 \%$ had mild symptoms, $18 \%$ were hospitalized, $6 \%$ admitted to the ICU, and $2.5 \%$ intubated. 

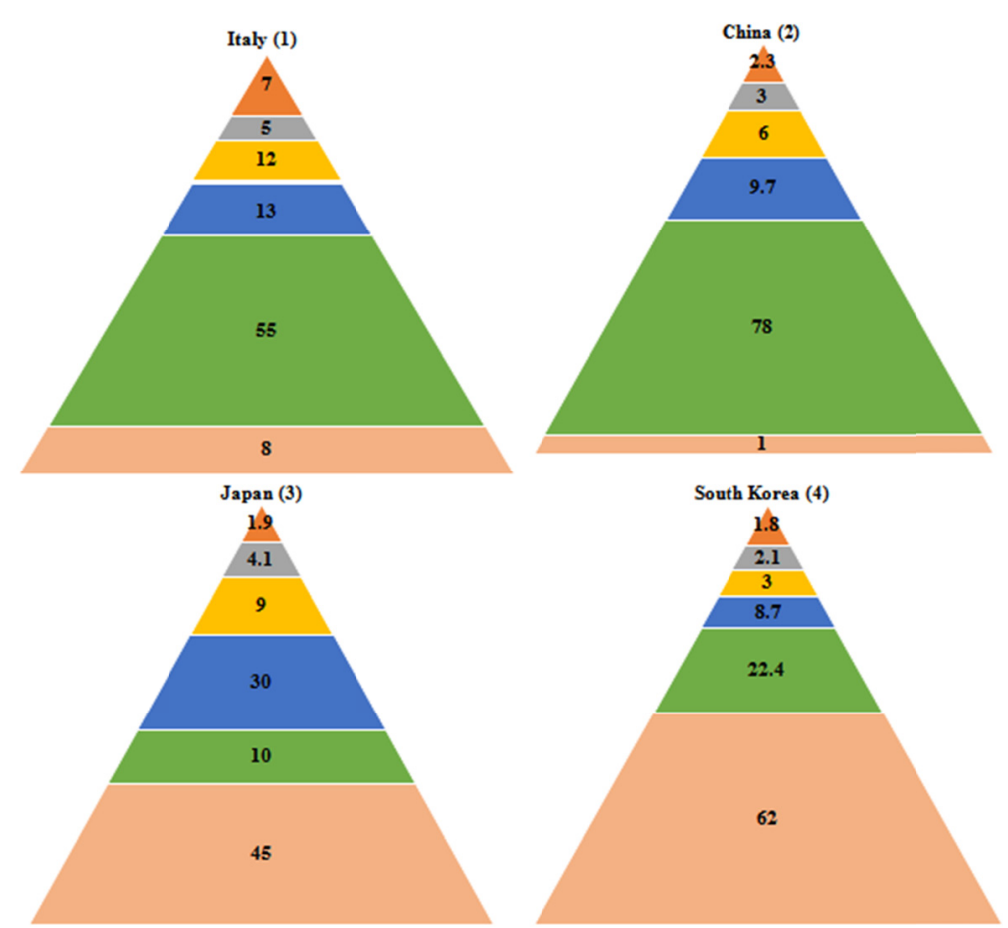

Fig. 2. The Italy (No. 1), China (No. 2), Japan (No. 3), and South Korea (No. 4) scenarios

\begin{tabular}{|c|c|c|c|c|c|c|c|}
\hline $\begin{array}{l}\text { Variables } \\
\text { Scenario }\end{array}$ & $\begin{array}{c}\text { No symptoms } \\
(\%)\end{array}$ & $\begin{array}{c}\text { Mild } \\
(\%)\end{array}$ & $\begin{array}{c}\text { Hospitalized } \\
(\%)\end{array}$ & $\begin{array}{l}\mathrm{ICU} \\
(\%)\end{array}$ & $\begin{array}{c}\text { Intubation } \\
(\%)\end{array}$ & $\begin{array}{l}\text { CFR } \\
(\%)\end{array}$ & References \\
\hline Scenario 1 & 8 & 55 & 13 & 12 & 5 & 7 & $(21)$ \\
\hline Scenario 2 & 1 & 78 & 9.7 & 6 & 3 & 2.3 & $(11,23)$ \\
\hline Scenario 3 & 45 & 10 & 30 & 9 & 4.1 & 1.9 & (24) \\
\hline Scenario 4 & 62 & 22.4 & 8.7 & 3 & 2.1 & 1.8 & $(25-27)$ \\
\hline Scenario 5 & 90 & 8 & 42 & 29 & 6 & 1.5 & $(56,57)$ \\
\hline Scenario 6 & 56.9 & 31 & 1.1 & 9.7 & 0.7 & 0.6 & $(31-33)$ \\
\hline Scenario 7 & 75 & 15 & 6 & 2.5 & 1 & 0.5 & $(34,35)$ \\
\hline
\end{tabular}

${ }^{a} \mathrm{CFR}$, case fatality rate; ICU, intensive care unit.

The fatality rate in this scenario was $1.5 \%$ (Fig. 3, Table 3).

The sixth scenario is related to the Diamond Princess cruise ship (31-33). The frequency of asymptomatic cases was $56.9 \%$ and the case fatality rate was $0.6 \%$. Also, ICU hospitalization in this scenario was $9.7 \%$ (Fig. 3 and Table 3).

Two studies (Simon P (35) and Stock, James H et al (34)) conducted in Iceland examined almost the entire population used in the ninth scenario (Table 2). In this scenario, $75 \%$ of cases were reported to be without clinical symptoms and other cases had mild symptoms, fewer patients were hospitalized and admitted to the ICU and intubation cases were much lower. The fatality rate was low because of the fact that fewer severe cases were observed, fewer deaths were reported, and the denominator was less (Fig. 3 and Table 3).

Finally, the clinical spectrum of COVID-19 in all scenarios are shown in Figure 4.

\section{Discussion}

In this research, 7 scenarios were considered for COVID-19, in which the range of fatality percentage was estimated to be $0.1 \%$ to $7 \%$, the range of asymptomatic cases was $1 \%$ to $90 \%$, the range of cases with moderate symptoms was $8 \%$ to $78 \%$, the range of ICU cases was $0.5 \%$ to $14.2 \%$, and finally the intubation percentage was estimated to be $0.2 \%$ to $12.2 \%$.

Asymptomatic infection at the time of laboratory confirmation has been reported in the results of various studies (36-39). Estimates were likely to be more accurate in cases where asymptomatic individuals were sampled, as a large proportion of cases that had symptoms were older people or people with underlying diseases. Based on the results of this study, in different studies, a range of about $1 \%$ to $90 \%$ of cases of COVID-19 were asymptomatic. Asymptomatic cases have been reported more in communities with more testing (40). The CFR is greatly subject to selection bias because given the policies of different countries, in many cases more severe cases have been tested $(39,41)$. The 7 proposed COVID-19 pandemic scenarios show a range of possible parameters based on different studies in different parts of the world. All parameter values are based on reported COVID-19 data.

In the Italian scenario, COVID-19 fatality was reported to be $7 \%$, which was the highest fatality rate among the various scenarios prepared in this study. The average age 


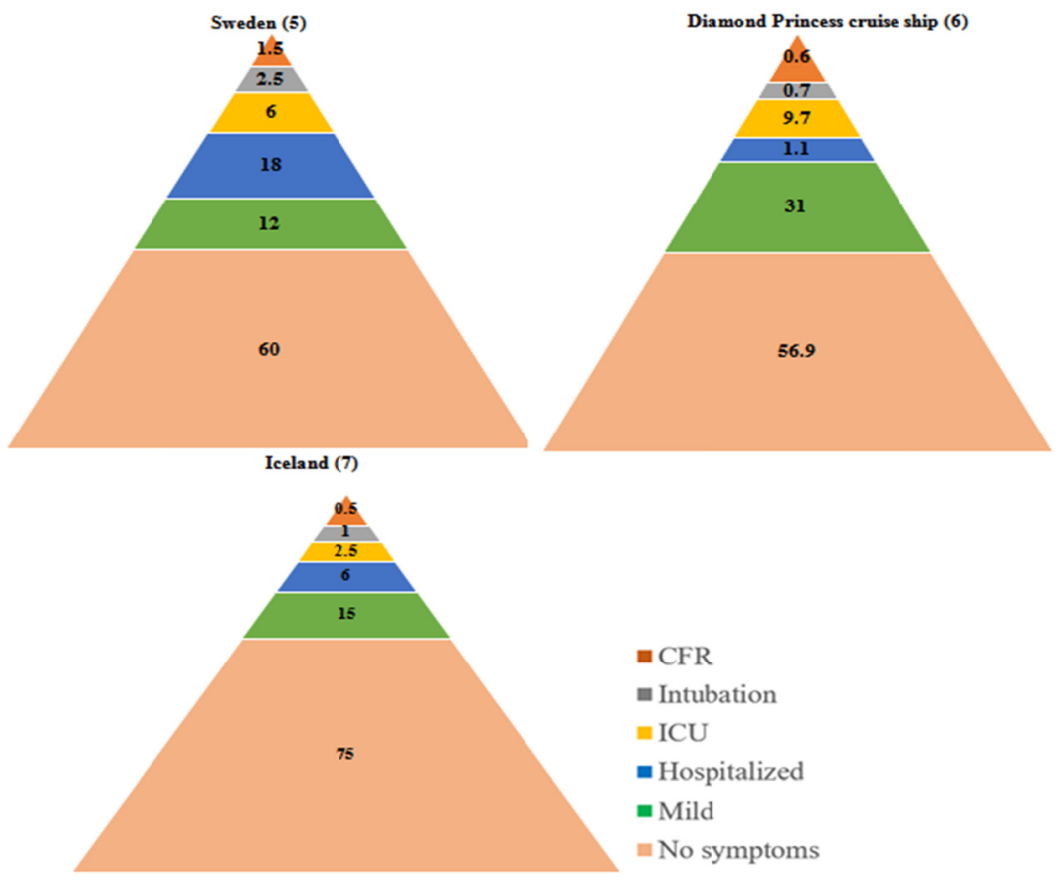

Fig. 3. The Sweden (No. 5), Diamond Princess cruise ship (No. 6), and Iceland (No. 7) scenarios

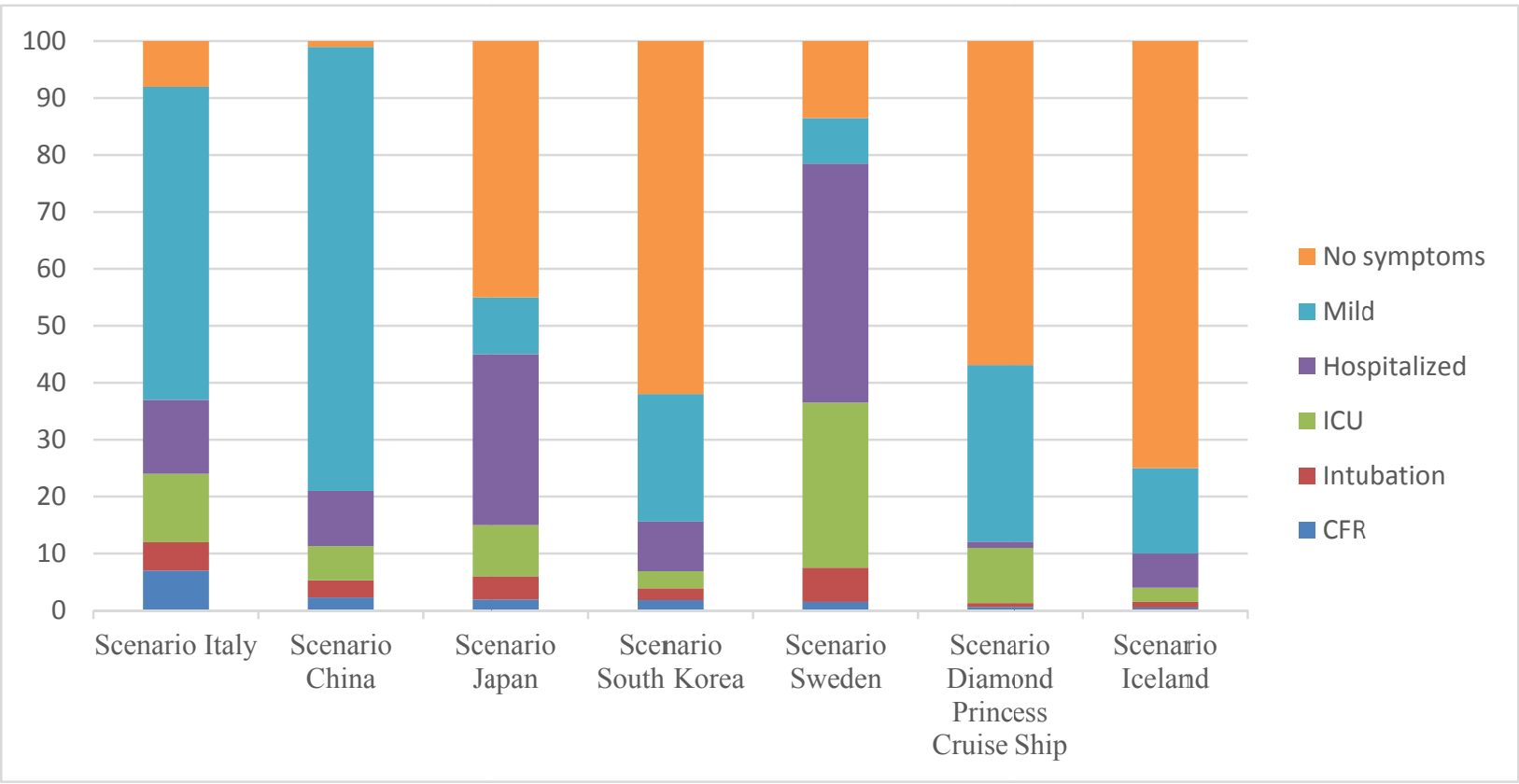

Fig. 4. Clinical Spectrum of COVID-19 in all Scenarios.

of Italy's population is higher than the world average. It is the second country in the world with the highest older population. The manner in which deaths are recorded also varies from country to country, and this difference may have a different effect on COVID-19 fatality rate reports. On the other hand, in the study conducted in Italy that was included in this review study, the identification of asymptomatic cases was not one of the main objectives $(21,42)$. Therefore, the results of Italy are not a good estimate for recognizing the iceberg of the disease, as it was conducted on symptomatic individuals, and because of the difference in the average age of the Itallian population and that of most countries in the world.

In the second scenario, which is related to China, the percentage of asymptomatic cases was $1 \%$, the percentage of cases with mild symptoms was $78 \%$, hospitalized cases were $9.7 \%$, intubated cases were $3 \%$, and CFR was $2.3 \%$. Although China carried out a rigid program for lockdown and controlled the disease acicording to official reports, more recent results show that China has made less effort than other countries, such as the United States and Italy, to identify and report asymptomatic or mild-symptom indi- 
viduals. Other reasons for the lack of access to all clinical spectrums in this country include the low accuracy of diagnostic tests or lack of sufficient specialized trained personnel at the beginning of the epidemic (43-45). The Chinese scenario is included in this study because it was the basis for intervention and action for long. With the new findings, however, it seems that this scenario is not consistent with the clinical spectrum of the disease, and it cannot be a good indicator of iceberg of the disease.

In the fourth and fifth scenarios, the percentage of asymptomatic cases was estimated to be $62 \%$ and $60 \%$, respectively. The fatality rate in these scenarios was estimated to be $1.8 \%$ and $1.5 \%$, respectively. These estimates in these scenarios indicate the presence of the virus among asymptomatic patients. This reinforced the hypothesis of transmission of the disease through asymptomatic cases and expanded the scope of control interventions (37). The results of the fourth (Japan) and the fifth (Sweden) scenarios showed that as the activity of different countries in identifying asymptomatic cases increases, the estimated percentage of hospitalization, ICU admission, intubation, and fatality rate decreases. These estimates are closer to the clinical spectrum of the disease. The results of these studies showed that the fatality rate of the disease is lower than what was reported in the initial studies. However, these studies also had limitations, such as low activity, to find all spectrums of the disease. Therefore, they cannot be considered as the best estimate of the iceberg of the disease.

In the sixth and seventh scenarios, the percentage of asymptomatic cases was estimated to be $56.9 \%$ and $75 \%$, respectively. The fatality rate in these 2 scenarios was estimated to be $<1 \%$. However, clinical ratios and fatality rates vary from study to study in different parts of the world, and these values should be interpreted with caution, as many factors affect these indicators. Nonetheless, these 2 scenarios have reported $<1 \%$ of fatality rate because of their extensive screening of the entire population, which has identified a higher proportion of asymptomatic and mild cases of the disease. In these scenarios, the denominator of the calculation includes mild cases, which was reported to be $31 \%$ and $15 \%$, respectively. These 2 scenarios are better for understanding the iceberg of the disease.

In this study, we introduce the sixth and seventh scenarios as the best ones for identifying the iceberg of the disease. In these scenarios, special attention should be paid to asymptomatic and mild-symptom cases for disease control.

In studies and countries where only people who needed hospitalization were investigated, the lower part of the iceberg was not identified. This can be misleading for policymakers, health care managers, physicians, and staff. In addition, paying too much attention to severe cases of the disease can cause more fear in people.

The high fatality rate in the first, second, and third scenarios indicates that the disease detection and isolation strategy, which was implemented in the early stages of COVID-19 pandemic as the most important way to deal with the disease in the developed countries, is currently less important as the number of infected cases increases. This strategy is appropriate and applicable only when the number of cases of the disease is small. As the number of cases increases, so does the number of asymptomatic cases. On the other hand, infected people may not refer to other designated treatment or screening centers and may prefer self-medication at home to referral to treatment centers. Now, the best solution for these countries is telemedicine, which both reduces the burden of patients' visits to hospitals and reduces the risk of illness to health workers. In this way, front-line forces fighting the disease remain safe and active and eventually gain access to all cases of the disease.

It is now known that, with the spread of the disease, a large proportion of cases are asymptomatic or mild. Health policymakers and professionals must prioritize identifying or accurately estimating the number of COVID-19 infections to control the disease and break the transmission chain to minimize asymptomatic individuals so that they can control and even monitor the disease in their communities $(11,46,47)$.

In addition, it should be noted that the clinical spectrum of the disease and CFR may lead to different estimates of the spectrum of the disease, depending on the demographic context of countries and even differences in the proportion of underlying diseases in different populations (48). Demographic variables, such as age, sex, marital status, and environmental variables may affect these estimates and CFR (49-51).

In some cases, the disease may have occurred in more high-risk groups, such as the older people or nursing homes. In such a scenario, there will be more severe cases and deaths. Some estimates in some communities may be due to these reasons $(52,53)$.

Since most deaths occur among people with underlying diseases, it is likely that all deaths will be attributed to COVID-19 (overestimation) and the role of the underlying diseases will be ignored. Cases of death from COVID-19 may even be considered and reported as death induced by other underlying diseases (undlerestimation). Therefore, it is important to pay attention to how deaths are recorded in different countries when the results and especially mortality rates are interpreted (52). Identifying the iceberg model and the clinical spectrum of the disease is of great importance in the epidemiology and natural history of the disease. This study is a quick review to identify the natural knowledge and clinical spectrum of COVID-19, taking into account the results of studies published in different countries. In addition, with the results of this study, basic steps can be taken towards the development of COVID-19 preventive measures.

One of the limitations of this study was the dispersion of the results of different studies, the inconsistency of study results and differences in the methods, examination and diagnosis policies, official reporting, and testing. In addition, the limited number of studies in each country and limited reports related to each of the indicators of fatality rate, hospitalization, ICU admission, intubation, and asymptomatic or mild-symptom cases prevented the performance of classical analyses, such as meta-analysis. 
Another limitation of the study may be that a number of cases in the study may have shown some symptoms in the later stages of the infection $(54,55)$.

\section{Conclusion}

There are currently various estimates of the clinical spectrum of COVID-19 in the world. Differences in estimates have confused health policymakers and managers, physicians, and other health care professionals. Understanding the clinical spectrum of this disease and the characteristics of its natural knowledge can be effective in developing interventions and prevention methods to tackle the disease transmission. The results of this study showed that with the increase in the ability of countries to identify COVID-19 patients, the number of asymptomatic cases increased, which can provide a more accurate estimate of the clinical spectrum of the disease from asymptomatic cases to the fatality rate of COVID-19. According to the results of the present study, we believe that scenarios of the Diamond Princess Cruise Ship and Iceland are closer to the reality of the clinical spectrum and natural knowledge of the disease. These 2 scenarios representing $0.6 \%$ and $0.5 \%$ of deaths, $0.7 \%$ and $1 \%$ of intubations, $2.5 \%$ and $9.7 \%$ of ICU admissions, $1.1 \%$ and $6 \%$ of hospitalizations, $15 \%$ and $31 \%$ of cases with mild symptoms, and finally $56.9 \%$ and $75 \%$ of asymptomatic cases of COVID-19, respectively-should now be considered as the basis for the natural knowledge of the disease. Understanding the clinical spectrum and natural knowledge of the disease and paying attention to asymptomatic or mild-symptom cases can help to make better decisions and develop more effective interventions to control the disease. To prove the results of this study and obtain more appropriate and accurate information about the clinical spectrum and iceberg of the disease, it is necessary to conduct a cohort study with a large statistical population.

\section{Acknowledgement}

This work was supported by Deputy of Research of Kurdistan University of Medical Sciences, which was recorded under the following ethical code: IR.MUK.REC.1399.005.

\section{Conflict of Interests}

The authors declare that they have no competing interests.

\section{References}

1. You C, Deng Y, Hu W, Sun J, Lin Q, Zhou F, et al. Estimation of the Time-Varying Reproduction Number of COVID-19 Outbreak in China. Available at SSRN 3539694. 2020.

2. Wu JT, Leung K, Leung GM. Nowcasting and forecasting the potential domestic and international spread of the 2019-nCoV outbreak originating in Wuhan, China: a modelling study. Lancet. 2020;395(10225):689-97.

3. Velavan TP, Meyer CG. The Covid-19 epidemic. Trop Med Int Health. 2020.

4. Organization WH. Coronavirus disease 2019 (COVID-19): situation report, 80. 2020.

5. Al-Tawfiq JA. Asymptomatic coronavirus infection: MERS-CoV and SARS-CoV-2 (COVID-19). Travel Med Infect Dis. 2020.
6. Vickers NJ. Animal communication: when i'm calling you, will you answer too? Curr Biol. 2017;27(14):R713-R5.

7. Michaels MG, La Hoz RM, Danziger Isakov L, Blumberg EA, Kumar D, Green M, et al. Coronavirus Disease 2019: Implications of Emerging Infections for Transplantation. Am J Transplant. 2020.

8. Linton NM, Kobayashi T, Yang Y, Hayashi K, Akhmetzhanov AR, Jung SM, et al. Epidemiological characteristics of novel coronavirus infection: A statistical analysis of publicly available case data. medRxiv. 2020.

9. Guan WJ, Ni ZY, Hu Y, Liang WH, Ou CQ, He JX, et al. Clinical characteristics of 2019 novel coronavirus infection in China. MedRxiv. 2020.

10. Wu Z, McGoogan JM. Characteristics of and Important Lessons From the Coronavirus Disease 2019 (COVID-19) Outbreak in China: Summary of a Report of 72314 Cases From the Chinese Center for Disease Control and Prevention. JAMA. 2020.

11. Wu Z, McGoogan JM. Characteristics of and important lessons from the coronavirus disease 2019 (COVID-19) outbreak in China: summary of a report of 72314 cases from the Chinese Center for Disease Control and Prevention. JAMA. 2020;323(13):1239-42.

12. Yao H, Chen JH, Xu YF. Patients with mental health disorders in the COVID-19 epidemic. Lancet Psychilatry. 2020;7(4):e21.

13. Yao X, Ye F, Zhang M, Cui C, Huang B, Niu P, et al. In Vitro Antiviral Activity and Projection of Optimized Dosing Design of Hydroxychloroquine for the Treatment of Severe Acute Respiratory Syndrome Coronavirus 2 (SARS-CoV-2). Clin Infect Dis. 2020.

14. Yang Y, Lu Q, Liu M, Wang Y, Zhang A, Jalali N, et al. Epidemiological and clinical features of the 2019 novel coronavirus outbreak in China. medRxiv. 2020

15. Surveillances V. The epidemiological characteristics of an outbreak of 2019 novel coronavirus diseases (COVID-19)-China, 2020. CCDC Weekly. 2020;2(8):113-22.

16. Cucinotta D, Vanelli M. WHO declares COVID-19 a pandemic. Acta Biomed. 2020;91(1):157-60

17. Organization WH. Coronavirus disease 2019 (COVID-19): situation report, 72. 2020.

18. Bai Y, Yao L, Wei T, Tian F, Jin DY, Chen L, et al. Presumed asymptomatic carrier transmission of COVID-19. JAMA. 2020;323(14):1406-7.

19. Magombedze G, Ngonghala CN, Lanzas C. Evalution of the "iceberg phenomenon" in Johne"s disease through mathematical modelling. PloS One. 2013;8(10):e76636.

20. Bhopal RS. Concepts of epidiemiology: integrating the ideas, theories, principles, and methods of epidemiology: Oxford University Press; 2016.

21. Balduzzi A, Brivio E, Rovelli A, Rizzari C, Gasperini S, Melzi ML, et al. Lessons after the early management of the COVID-19 outbreak in a pediatric transplant and hemato-oncology center embedded within a COVID-19 dedicated hospital in Lombardia, Italy. Bone Marrow Transplant. 2020:1-6

22. Report NI. Integrated surveillance of COVID-19 in Italy https://www.epicentro.iss.it/coronavirus/bollettino/Infografica_17marz o\%20ENG.pdf

23. Guan WJ, Ni ZY, Hu Y, Liang WH, Ou CQ, He JX, et al. Clinical characteristics of coronavirus disease 2019 in China. N Engl J Med. 2020;382(18):1708-20.

24. Russell TW, Hellewell J, Jarvis CI, Van Zandvoort K, Abbott S, Ratnayake R, et al. Estimating the infection and case fatality ratio for coronavirus disease (COVID-19) using age-adjusted data from the outbreak on the Diamond Princess cruise ship, February 2020. Eurosurveillance. 2020;25(12):20001256.

25. Jung CY, Park H, Kim DW, Choi YJ, Kim SW, Chang TI. Clinical characteristics of asymptomatic patients with COVID-19: a nationwide cohort study in South Korea. Int J Infect Dis. 2020;99:2668.

26. Oh J, Lee JK, Schwarz D, Ratcliffe HL, Markuns JF, Hirschhorn LR. National response to COVID-19 in the Republic of Korea and lessons learned for other countries. Health Siyst Reform. 2020;6(1):e-1753464.

27. Cho SI, Yoon S, Lee HJ. Impact of comorbidity burden on mortality in patients with COVID-19: a retrospective analysis of the Korean health insurance database. 2020.

28. Sjödin H, Johansson AF, Brännström Å, Farooq Z, Kriit HK, Wilder-Smith A, et al. Covid-19 health care demand and mortality in Sweden in response to non-pharmaceutical (NPIs) mitigation and suppression scenarios. MedRxiv https://doi org/101101/202003. 
2020;20.

29. Keeley AJ, Evans CM, De Silva TI. Asymptomatic SARS-CoV-2 infection: the tip or the iceberg? Thorax. 2020;75(8):621-2.

30. Moriarty LF. Public health responses to COVID-19 outbreaks on cruise ships-worldwide, February-March 2020. MMWR Morb Mortal Wkly Rep. 2020;69.

31. Yamagishi T, Kamiya H, Kakimoto K, Suzuki M, Wakita T. Descriptive study of COVID-19 outbreak among passengers and crew on Diamond Princess cruise ship, Yokohama Port, Japan, 20 January to 9 February 2020. Eurosurveillance. 2020;25(23):2000272.

32. Russell T, Hellewell J, Jarvis C, Van-Zandvoort K, Abbott S, Ratnayake R, et al. Estimating the infection and case fatality ratio for COVID-19 using age-adjusted data from the outbreak on the Diamond Princess cruise ship. medrxiv. 2020 Mar 5.

33. Yamahata Y, Shibata A. Preparation for Quarantine on the Cruise Ship Diamond Princess in Japan due to COVID-19. J Med Int Res. 2020;6(2):e18821.

34. Stock JH, Aspelund KM, Droste M, Walker CD. Estimates of the undetected rate among the sars-cov-2 infected using testing data from iceland. medRxiv. 2020.

35. Simon P. Robust Estimation of Infection Fatality Rates during the Early Phase of a Pandemic. medRxiv. 2020.

36. Lauer SA, Grantz KH, Bi Q, Jones FK, Zheng Q, Meredith HR, et al. The incubation period of coronavirus disease 2019 (COVID-19) from publicly reported confirmed cases: estimation and application. Ann. Intern Med. 2020;172(9):577-82.

37. Mizumoto K, Kagaya K, Zarebski A, Chowell G. Estimating the asymptomatic proportion of coronavirus disease 2019 (COVID-19) cases on board the Diamond Princess cruise ship, Yokohama, Japan, 2020. Eurosurveillance. 2020;25(10):2000180.

38. Ki M. Epidemiologic characteristics of early cases with 2019 novel coronavirus (2019-nCoV) disease in Korea. Epidemiol Health. $2020 ; 42$.

39. Novel CPERE. The epidemiological characteristics of an outbreak of 2019 novel coronavirus diseases (COVID-19) in China. Zhonghua Liu Xing Bing Xue Za Zhi. 2020;41(2):145.

40. Hoehl S, Rabenau H, Berger A, Kortenbusch M, Cinatl J, Bojkova $\mathrm{D}$, et al. Evidence of SARS-CoV-2 infection in returning travelers from Wuhan, China. N Engl J Med. 2020;382(13):1278-80.

41. Mahase E. Coronavirus: covid-19 has killed more people than SARS and MERS combined, despite lower case fatality rate. BMJ. 2020.

42. Onder G, Rezza G, Brusaferro S. Case-fatality rate and characteristics of patients dying in relation to COVID-19 in Italy. JAMA. 2020.

43. Mission W-CJ. Report of the WHO-China Joint Mission on Coronavirus Disease 2019 (COVID-19). 2020;(16-24 February).

44. Organization WH. Modes of transmission of virus causing COVID19: implications for IPC precaution recommendations: scientific brief, 27 March 2020. World Health Organization; 2020.

45. Organization WH. Report of the WHO-China Joint Mission on Coronavirus Disease 2019 (COVID-19), 16-24 February 2020 (2020).

46. O'Grady NP, Alexander M, Dellinger EP, Gerberding JL, Heard SO, Maki DG, et al. Guidelines for the prevention of intravascular catheter-related infections. The Hospital Infection Control Practices Advisory Committee, Center for Disease Control and Prevention, US. Pediatrics. 2002;110(5):e51-e.

47. Lo IL, Lio CF, Cheong HH, Lei CI, Cheong TH, Zhong X, et al. Evaluation of SARS-CoV-2 RNA shedding in clinical specimens and clinical characteristics of 10 patients with COVID-19 in Macau. Int J Biol Sci. 2020;16(10):1698.

48. Abdollahi E, Champredon D, Langley JM, Galvani AP, Moghadas SM. Temporal estimates of case-fatality rate for COVID-19 outbreaks in Canada and the United States. CMAJ. 2020.

49. Dudel C, Riffe T, Acosta E, van Raalte AA, Myrskyla M. Monitoring trends and differences in COVID-19 case fatality rates using decomposition methods: Contributions of age structure and agespecific fatality. medRxiv. 2020.

50. Rajgor DD, Lee MH, Archuleta S, Bagdasarian N, Quek SC. The many estimates of the COVID-19 case fatality rate. Lancet Infect Dis. 2020.

51. Del Rio C, Malani PN. COVID-19-new insights on a rapidly changing epidemic. JAMA. 2020;323(14):1339-40.

52. Vincent J-L, Taccone FS. Understanding pathways to death in patients with COVID-19. Lancet Respir Med. 2020;8(5):430-2.

53. Emanuel E, Persad G, Upshur R. Allocation of scarce medical resources in the time of Covid-19 [published online ahead of print 2020]. N Engl J Med. 2020.

54. Cereda D, Tirani M, Rovida F, Demicheli V, Ajelli M, Poletti P, et al. The early phase of the COVID-19 outbreak in Lombardy, Italy. arXiv preprint arXiv:200309320. 20120.

55. Luo SH, Liu W, Liu ZJ, Zheng XY, Hong CX, Liu ZR, et al. A confirmed asymptomatic carrier of 2019 novel coronavirus. Chin Med J. 2020;133(9):1123-5. 